\title{
ANÁLISE NUMÉRICA DA INFLUÊNCIA DA GEOMETRIA DO CAPILAR DE INJEÇÃO NO PROCESSO ANTISSOLVENTE SUPERCRÍTICO (SAS)
}

\author{
R. A. ALMEIDA ${ }^{1}$, R. V. P. REZENDE ${ }^{2}$, H.F. MEIER, ${ }^{3}$ D. NORILER ${ }^{3}$, V.F. CABRAL ${ }^{1}$ e L. \\ CARDOZO-FILHO ${ }^{1}$, \\ ${ }^{1}$ Universidade Estadual de Maringá, Departamento de Engenharia Química \\ ${ }^{2}$ Universidade Federal de Santa Catarina, Departamento de Engenharia Química \\ ${ }^{3}$ Universidade Regional de Blumenal , Departamento de Engenharia Química \\ E-mail para contato: regiani_al@hotmail.com
}

\begin{abstract}
RESUMO - Processos como o SAS (Supercritical Antisolvent) que empregam CO2 em estado supercrítico como antissolvente permitem a precipitação de uma variedade de produtos em escala nanométrica da indústria farmacêutica e de alimentos. Pouco ainda se conhece à respeito do impacto dos parâmetros do processo como a geometria do capilar de injeção no tamanho e forma das partículas precipitadas. Um modelo matemático foi proposto e o código ANSYS FLUENT foi empregado para a sua solução. Em uma aproximação bidimensional, capilares de dois comprimentos e dois diâmetros distintos foram testados a fim de verificar sua influência no comportamento fluidodinâmico da mistura supercrítica e consequentemente no tamanho e na forma das partículas cristalizadas. A partir da análise das variáveis velocidade, intensidade de turbulência e variância da fração de mistura observou-se que a configuração do tubo capilar de menor diâmetro e maior comprimento oferece condições para um processo de mistura mais eficiente.
\end{abstract}

\section{INTRODUÇÃO}

Um fluido se encontra na condição supercrítica quando se encontra à temperatura e pressão acima das referidas como o seu ponto crítico (Jerzy et al., 2004). Existem atualmente várias técnicas de precipitação que utilizam tecnologia supercrítica, em particular a técnica SAS (supercritical Antisolvent), considerada aqui é usada quando o soluto de interesse não é solúvel no fluido supercrítico mas sim em um solvente orgânico. A mistura contendo o soluto e o solvente orgânico entra através de um tubo capilar em uma câmara de precipitação pressurizada e previamente carregada com antissolvente, interagindo com o antissolvente (em condições supercríticas) o qual continua a ser injetado pelo mesmo capilar de forma coaxial, elevando a difusão do antissolvente na mistura orgânica e provocando a precipitação do soluto (De Marco and Reverchon, 2011, Franceschi et al., 2008, Rossmann et al., 2012).

O entendimento dos mecanismos de precipitação envolvidos no processo SAS requerem uma boa fundamentação termodinâmica, fluidodinâmica, de transferência de massa, de 


\section{9 a 22 de outubro de 2014 \\ Florianópolis/SC}

nucleação e de cescimento de partículas. Tais mecanismos são dependentes de parâmetros de operação e ainda não foram bem estudados do ponto de vista experimental resultando em grandes discrepâncias em termos de tamanho e forma das partículas como observado por Tenorio et al. (2007). A exploração experimental do processo em ampla gama de condições de operação resulta em um procedimento dispendioso e caro, assim, a análise numérica a partir da fluidodinâmica computacional (CFD) é uma boa alternativa para a determinação de condições favoráveis à precipitação de partículas em escala nanométrica. Vale ressaltar que a modelagem completa deste processo é complexa pela ocorrência de diferentes fenômenos em diferentes escalas e que, e na maioria dos trabalhos de pesquisa têm sido considerados apenas alguns dos fenômenos envolvidos no processo (Cardoso et al., 2008, Erriguible et al., 2013a, Reverchon et al., 2008) .

Ao longo da última década, a recristalização de drogas, produtos da indústria alimentícia e biopolímeros empregando $\mathrm{CO}_{2}$ como antissolvente esteve voltada para à quantificação da influência de parâmetros de processo sobre a morfologia e o tamanho do produto final com referência especial aos parâmetros temperatura e pressão de operação, vazões de entrada de solvente e de antissolvente (Erriguible et al., 2013b). Menor atenção tem sido voltada à influência da geometria da câmara de precipitação tal como as dimensões do tubo capilar de injeção. Segundo Sierra-Pallares (2012) que em seu trabalho numérico emprega capilares de dois diâmetros distintos, relata que uma diminuição no diâmetro do capilar modifica completamente o padrão do escoamento na câmara promovendo uma mistura mais rápida e eficiente. A utilização de capilar de maior comprimento possibilitando considerar a injeção de $\mathrm{CO}_{2}$ em um ponto específico da câmara foi considerada por Cardoso et al. (2008) e pode diminuir zonas mortas e melhorar a qualidade da mistura, resultando em uma distribuição de tamanhos de partículas mais estreita. Os trabalhos apresentados pela literatura e que tratam da geometria do capilar de injeção sugerem estudos mais amplos em uma gama maior de condições.

Assim, neste trabalho é apresentado um estudo numérico sobre a influência das dimensões do capilar de injeção nos padrões do escoamento e no processo de mistura na câmara SAS. A modelagem matemática é considerada sob a hipótese de escoamento estacionário, turbulento, não-isotérmico e em uma única fase (em condições de temperatura e pressão acima do ponto crítico da mistura). Serão considerados na mistura o $\mathrm{CO}_{2}$ como antissolvente e o etanol como solvente orgânico sem a presença de um soluto dissolvido.

\section{MODELAGEM MATEMÁTICA}

\subsection{Modelagem Termodinâmica}

Foi empregada a equação de estado de Peng-Robinson para a determinação da densidade do sistema binário considerado: 
$P=\frac{R T}{v-b_{m}}-\frac{a_{m}}{v\left(v+b_{m}\right)+b_{m}\left(v-b_{m}\right)}$,

onde $P$ é a pressão, $v$ o volume molar, $T$ a temperatura, $a_{m}$ e bm, as constantes da mistura e para o seu cálculo considerou-se a regra de mistura quadrática de van der Waals Poling et al. (2004) e regras de combinação: $a_{i j}=\sqrt{a_{i} a_{j}}\left(1-k_{i j}\right)$ e $b_{i j}=\left(b_{i}+b_{j}\right)\left(1-l_{i j}\right) / 2$, com parâmetros de interação binária $\mathrm{k}_{i i}=\mathrm{l}_{i i}=0, \mathrm{k}_{i j}=0,097$ e $\mathrm{l}_{i j}=-0,000966$ para o sistema $\mathrm{CO}_{2}$ e etanol (Cardoso et al., 2008).

\subsection{Equações de conservação}

O modelo de turbulência $k-\varepsilon$ foi empregado com a técnica de média ponderada pela massa (média de Favre), assim, uma variável média local $\tilde{\phi}$ é definida como $\tilde{\phi}=\overline{\rho \phi} / \bar{\rho}$. Onde o símbolo "barra" denota a média de Reynolds. Assim o campo de escoamento para uma variável $\phi$ qualquer, pode ser decomposto como: $\phi=\tilde{\phi}+\phi$ " e, trocando as variáveis, por seus equivalentes valor médio e flutuação (Wilcox, 1993), obtém-se as equações médias de conservação: da continuidade; da quantidade de movimento; da energia; da fração de mistura e sua variância respectivamente:

$\partial_{i}\left(\bar{\rho} \tilde{u}_{i}\right)=0$

$\partial_{\mathrm{i}}\left(\bar{\rho} \tilde{u}_{i} \tilde{u}_{j}\right)=\partial_{j} \bar{P}+\partial_{j}\left(\tilde{\tau}_{i j}-\bar{\rho} \widehat{u_{i}^{\prime} u_{j}^{\prime}}\right)$

$\partial_{i}\left(\bar{\rho} \tilde{u}_{i} \tilde{h}\right)=\partial_{j}\left\{\left[\left(K_{m}+K_{T}\right) / c_{p}\right] \partial_{j} \tilde{h}\right\}+\tilde{\tau}_{i j} \partial_{j} \tilde{u}_{i}$

$\partial_{j}\left(\bar{\rho} \tilde{u}_{j} \tilde{f}\right)=\partial_{j}\left[\bar{\rho}\left(D_{m}+D_{T}\right) \partial_{j} \tilde{f}\right]$

$\partial_{j}\left(\bar{\rho} \tilde{u}_{j} \tilde{\sigma}^{2}\right)=\partial_{j}\left[\bar{\rho}\left(D_{m}+D_{T}\right) \partial_{j} \tilde{\sigma}^{2}\right]+2 D_{T}\left(\partial_{j} \tilde{f}\right)^{2}-2 \frac{\varepsilon}{k} \tilde{\sigma}^{2}$,

onde o termo $-\bar{\rho} \overline{u_{i}^{\prime} u_{j}^{\prime}}$ é o tensor tensão de Reynolds, resolvido pelo modelo de turbulência, e $\tilde{\tau}_{i j}$ o tensor tensão viscoso (é considerado fluido Newtoniano), onde $\tilde{u}_{i}, i \in\{1,2,3\}$ são as componentes médias do vetor velocidade; $\tilde{h}$ é a entalpia total, $K_{m}$ e $K_{T}$ são a condutividade térmica molecular e condutividade térmica turbulenta, respectivamente e $c_{p}$ o calor específico da mistura. Onde $D_{T}=\mu_{T} / \bar{\rho} S c_{T}, S c_{T}$ é o número de Schmidt turbulento e $D_{m}$ é a difusividade molecular. A fração de mistura é interpretada aqui como a fração mássica de fluido alimentado no sistema a partir da entrada de solução $f=\left(y_{C o 2}-y_{\alpha, C o 2}\right) /\left(y_{\alpha, \text { sol }}-y_{\alpha, C o 2}\right)$ (Henczka and Shekunov, 2005), sendo $y_{\mathrm{Co2}_{2}}$ a fração mássica de $\mathrm{CO} 2, y_{\alpha, c o 2}$ a fração mássica de $\mathrm{CO}_{2}$ na entrada de $\mathrm{CO}_{2}$ e $y_{\alpha, \text { sol }}$ a fração mássica de $\mathrm{CO}_{2}$ na entrada de solução respectivamente. E a variância da fração de mistura $\sigma^{2}$ representa o desvio da idealidade e o processo de mistura pode ser interpretado como uma dissipação desta variância (Jerzy et al., 2004). A viscosidade da 
mistura foi dada por: $\mu_{m}=\exp \left(y_{C_{2}} \ln \left(\mu_{E \operatorname{tanol}}\right)+y_{E \operatorname{tanol}} \ln \left(\mu_{C_{2}}\right)\right)$, onde $\mu_{E t a n o l}=8,34 \mathrm{e}^{-4}$ Pa.s $=\mathrm{e}$ $\mu_{\mathrm{Co} 2}=2 \mathrm{e}^{-5}$ Pa.s, onde $y_{\text {Etanol }}$ é a fração mássica de etanol.

\section{DOMÍNIO COMPUTACIONAL E PROCEDIMENTO NUMÉRICO}

A câmara SAS é um cilindro de 120mm de comprimento e $80 \mathrm{~mm}$ de diâmetro (Almeida et al., 2012) acoplada a um capilar de injeção coaxial. Foram simulados quatro casos a partir de um domínio computacional composto da geometria bidimensional e simetria axial: capilar de 20mm e $98 \mathrm{~mm}$ de comprimento com $100 \mu \mathrm{m}$ de diâmetro; e capilar de $98 \mathrm{~mm}$ de comprimento com $100 \mu \mathrm{m}$ e $240 \mu \mathrm{m}$ de diâmetro. As malhas hexaédricas têm aproximadamente 115,5x10 ${ }^{3}$ elementos como mostra a Figura 1. O software ANSYS FLUENT 13.0 foi utilizado para a solução numérica do sistema de equações. O sistema linearizado foi resolvido de forma segregada usando o solver SIMPLE para o acoplamento pressão-velocidade e esquemas de discretização espacial de primeira ordem. Como condições de contorno: vazão mássica para as entradas de antissolvente e de solução; de pressão nula na saída da câmara; de livre deslizamento nas paredes e simetria de eixo na simetria. A equação de estado (Equação (1)), bem como as Equações (5) e (6) foram implementadas via sub-rotinas em linguagem C.

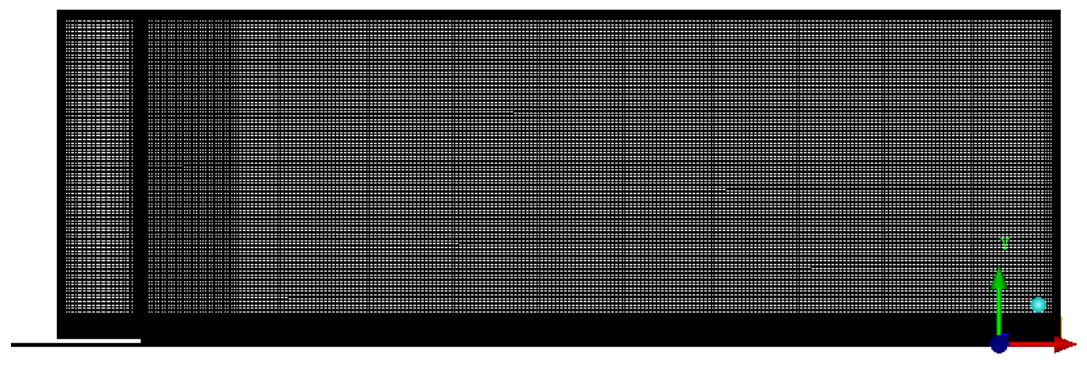

Figura 1 - Malha hexaédrica da câmara SAS acoplada ao capilar de 20mm de comprimento e $100 \mu \mathrm{m}$ de diâmetro.

A pressão e a temperatura de operação foram de 130bar e 313,15K respestivamente. A vazão mássica na entrada $\mathrm{CO}_{2}$ foi de $6,63 \times 10^{-4} \mathrm{~kg} / \mathrm{s}$ e a vazão mássica na entrada de etanol foi de $1,66 \times 10^{-5} \mathrm{~kg} / \mathrm{s}$.

\section{RESULTADOS}

Quanto a validação do modelo, a abertura do jato foi de aproximadamente $12^{\circ}$ para todos os casos o que está de acordo com aquela predita pela solução analítica de abertura para jatos turbulentos, apresentando também perfis gaussianos de concentração de espécies e de velocidade, como reportado por Cushman-Roisin (2013). A Figura 2 ilustra a abertura do jato e os perfis gaussianos de velocidade para dois dos casos simulados.

\subsection{Influência do Comprimento do Capilar}


No caso do capilar de menor comprimento com diâmetro $100 \mu \mathrm{m}$, o escoamento da mistura apresenta menor velocidade no interior da câmara $(4,0 \mathrm{~m} / \mathrm{s})$, do que quando comparada com valores da magnitude da velocidade para o capilar de mesmo diâmetro e $98 \mathrm{~mm}(4,8 \mathrm{~m} / \mathrm{s})$.
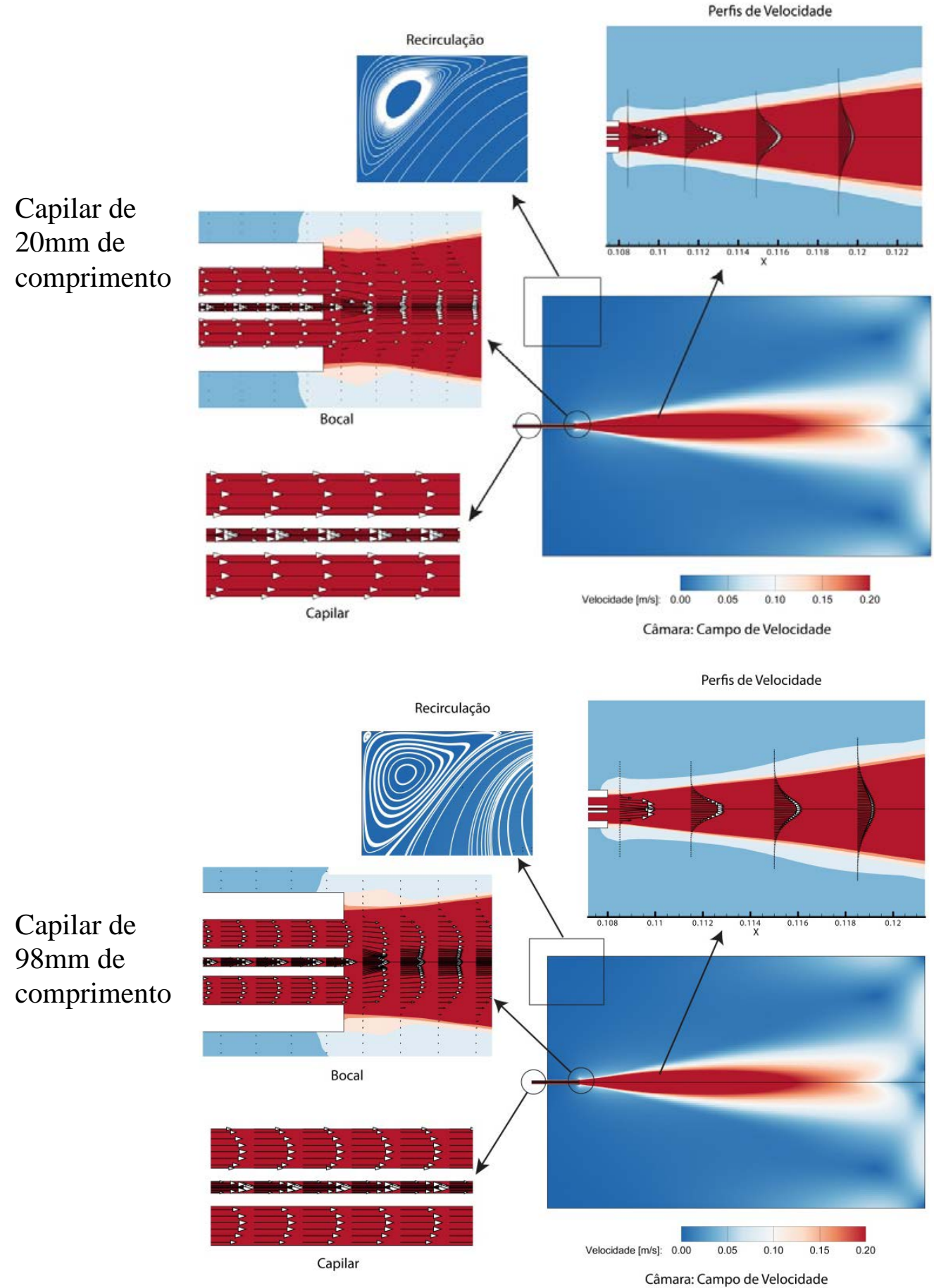

Figura 2 - Padrão de escoamento e perfil de velocidade para os dois comprimentos de capilares. 
Os padrões do escoamento mostraram um maior achatamento das linhas de corrente no sentido axial quando utilizado o capilar de maior comprimento, gerando neste caso mais zonas de recirculação próximas à junção da parede lateral com a tampa como se compara nas Figura 2 e na Figura 3. Porém, houve neste caso menores valores da variância da fração de mistura evidenciando uma mistura mais eficiente.

As maiores variações observadas quanto ao comprimento do capilar foram nas distribuições de densidade (os valores da densidade são maiores no interior da câmara com capilar de $20 \mathrm{~mm}$ ao longo da linha de centro da câmara, aproximadamente $140 \mathrm{~kg} / \mathrm{m}^{3}$ a mais) e na intensidade de turbulência. Houve um aumento de $5 \%$ na intensidade de turbulência no interior da câmara com o capilar de maior comprimento, aumentando a dispersão, melhorando o processo de mistura. Observou-se ainda para o capilar de maior comprimento linhas de corrente rápidas melhores distribuídas.

\subsection{Influência do Diâmetro do Capilar}

Observou-se no caso do capilar de menor diâmetro, maior velocidade na saída do capilar, maior intensidade de turbulência, menores valores na variância da fração de mistura bem como maior região rica em $\mathrm{CO}_{2}$ (cerca de duas vezes a mais do que no caso com o capilar de maior diâmetro), a Figura 3 mostra as distribuições de etanol para os dois casos. Este conjunto de fatos sugere um melhor processo de mistura entre as espécies na câmara SAS, mas com o capilar de $240 \mu \mathrm{m}$ também se obteve menos recirculações no interior da câmara, de modo que neste caso pode-se obter menor número de aglomerados dependendo do material a ser precipitado.
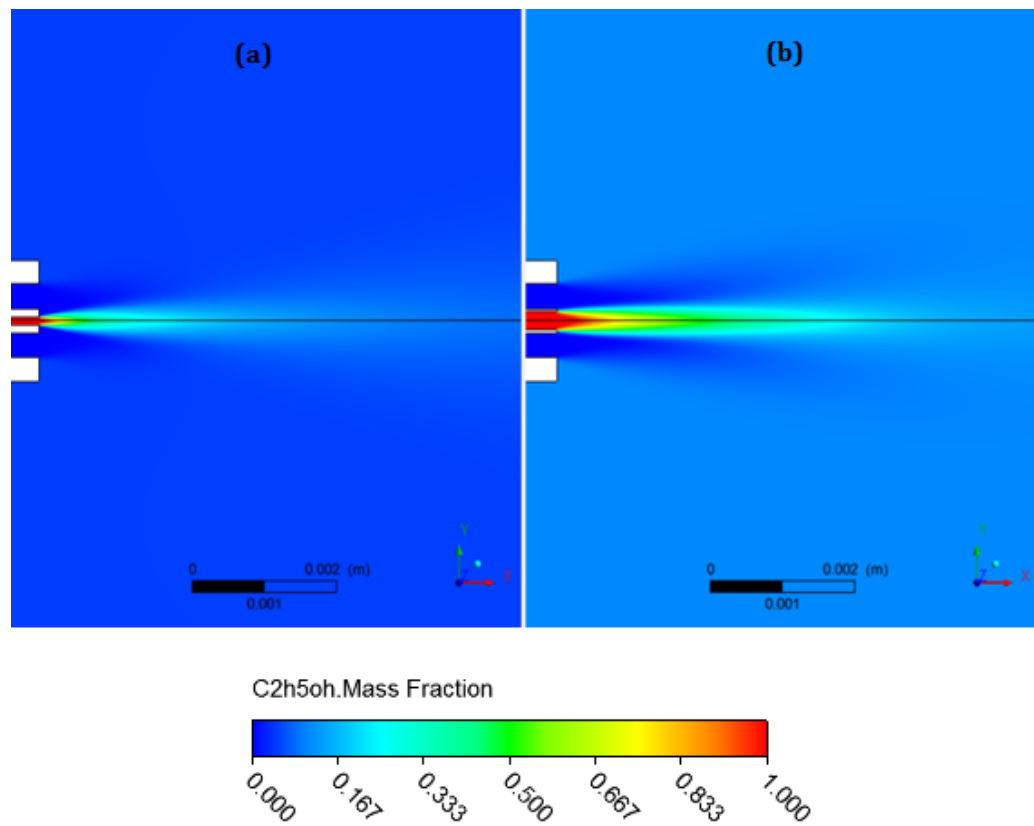

Figura 3- Perfis de fração mássica de etanol na região da saída do capilar:

(a) Capilar de $100 \mu \mathrm{m}$, (b) Capilar de $240 \mu \mathrm{m}$. 
Ainda, com o capilar de $100 \mu m$ há menor variação temperatura $(1,5 \mathrm{~K})$ até aproximadamente $3 \mathrm{~mm}$ na saída do capilar, após esta posição a temperatura exede àquela observada para o capilar de $240 \mu \mathrm{m}$ em aproximadamente $2 \mathrm{~K}$ pela linha de centro da câmara. Adimitindo-se que na região da saída do capilar há maior probabilidade de nucleação (Petit-Gas et al., 2009), isto pode ser levado em consideração na precipitação de compostos sensíveis à variações de temperatura e neste caso o capilar de menor diâmetro deve ser preferido.

\section{CONCLUSÕES}

Uma câmara de precipitação SAS foi modelada matematicamente a fim de obter informações a respeito do processo de mistura supercrítica quando se varia o comprimento e o diâmetro do capilar de injeção de $\mathrm{CO}_{2}$ e etanol. Os resultados demonstram boa concordância com a literatura quanto à abertura do jato e os perfis gaussianos de velocidade.

A partir da hipótese de que na saída do capilar inicia-se o processo de nucleação de partículas, a melhor combinação de parâmetros para se operar o processo obtendo uma mistura mais eficiente inclusive quando se considera a precipitação de solutos termosensíveis foi a utilização do capilar de maior comprimento e de menor diâmetro.

Assim, observou-se que os parâmetros: comprimento e diâmetro do capilar influem diretamente na dinâmica do escoamento corroborando a afirmação de Sierra-Pallares et al. (2012) e consequentemente nos mecanismos de precipitação, então análises como as apresentadas aqui podem ser realizadas antes de proceder com o procedimento experimental visto que de posse do modelo matemático, as simulações irão requerer pouco tempo computacional (em torno de 8 horas em um computador notebook ${ }^{1}$ ) e baixo custo na busca por condições que promovam uma boa mistura na câmara.

\section{REFERÊNCIAS}

ALMEIDA, R. A., REZENDE, R. V. D. P., MEIER, H. F., CABRAL, V. F., NORILER, D., FILHO, L. C. \& GUIRARDELLO, R. 2012. Modelagem Fluidodinâmca e Solução Numérica do Escoamento de Fluido Supercrítico Aplicados à Produção de Nanopartículas. XIX Congresso Brasileiro de Engenharia Química. Búzios-RJ.

CARDOSO, M. A. T., CABRAL, J. M. S., PALAVRA, A. M. F. \& GERALDES, V. 2008. CFD analysis of supercritical antisolvent (SAS) micronization of minocycline hydrochloride. The Journal of Supercritical Fluids, 47, 247-258.

CUSHMAN-ROISIN, B. 2013. Environmental Fluid Mechanics, United States of America, John Wiley \& Sons, Inc.

1 Processador: Intel(R) Core(TM) i5-2450M CPU @2.5GHz com velocidade de 2.50 GHz, memória (RAM) de 4Gb com sistema operacional Windows 7, 64 bits. 


\section{9 a 22 de outubro de 2014 \\ Florianópolis/SC}

DE MARCO, I. \& REVERCHON, E. 2011. Influence of pressure, temperature and concentration on the mechanisms of particle precipitation in supercritical antisolvent micronization. The Journal of Supercritical Fluids, 58, 295-302.

ERRIGUIBLE, A., FADLI, T. \& SUBRA-PATERNAULT, P. 2013a. A complete 3D simulation of a crystallization process induced by supercritical $\mathrm{CO} 2$ to predict particle size. Computers \& Chemical Engineering, 52, 1-9.

ERRIGUIBLE, A., LAUGIER, S., LATÉ, M. \& SUBRA-PATERNAULT, P. 2013b. Effect of pressure and non-isothermal injection on re-crystallization by $\mathrm{CO} 2$ antisolvent: Solubility measurements, simulation of mixing and experiments. The Journal of Supercritical Fluids, 76, 115-125.

FRANCESCHI, E., DE CESARO, A. M., FEITEN, M., FERREIRA, S. R. S., DARIVA, C., KUNITA, M. H., RUBIRA, A. F., MUNIZ, E. C., CORAZZA, M. L. \& OLIVEIRA, J. V. 2008. Precipitation of $\beta$-carotene and PHBV and co-precipitation from SEDS technique using supercritical CO2. The Journal of Supercritical Fluids, 47, 259-269.

HENCZKA, M. B., JERZY. \& SHEKUNOV, B. Y. 2005. Particle formation by turbulent mixing with supercritical antisolvent. Chemical Engineering Science, 60, 2193- 2201.

JERZY, BORIS, S., MAREK, H. \& BALDYGA 2004. Fluid Dynamics, Mass Transfer, and Particle Formation in Supercritical Fluids. Supercritical Fluid Technology for Drug Product Development. Informa Healthcare.

PETIT-GAS, T., BOUTIN, O., RASPO, I. \& BADENS, E. 2009. Role of Hidrodynamics in Supercritical Antisolvent Process. The Journal of Supercritical Fluids, 7.

POLING, B. E., PRAUSNITZ, J. M. \& O’CONNELL, J. P. 2004. The Properties of Gases and Liquids, McGraw-Hill Companies.

REVERCHON, E., ADAMI, R., CAPUTO, G. \& DE MARCO, I. 2008. Spherical microparticles production by supercritical antisolvent precipitation: Interpretation of results. The Journal of Supercritical Fluids, 47, 70-84.

ROSSMANN, M., BRAEUER, A., DOWY, S., GALLINGER, T. G., LEIPERTZ, A. \& SCHLUECKER, E. 2012. Solute solubility as criterion for the appearance of amorphous particle precipitation or crystallization in the supercritical antisolvent (SAS) process. The Journal of Supercritical Fluids, 66, 350-358.

SIERRA-PALLARES, J., MARCHISIO, D. L., PARRA-SANTOS, M. T., GARCÍA-SERNA, J., CASTRO, F. \& COCERO, M. J. 2012. A computational fluid dynamics study of supercritical antisolvent precipitation: Mixing effects on particle size. AIChE Journal, 58, 385-398.

TENORIO A., G. M. D., PEREYRA C.M., MARTINEZ DE LA OSSA E.J. 2007. Relative importance of the operating conditions involved in the formation of nanoparticles of ampicillin by supercritical antisolvent precipitation. Ind. Eng. Chem., 46.

WILCOX, D. C. 1993. Turbulence Modeling for CFD La Canada, California DCW Industries. 